\title{
EECBcast: Pharaonis Approach based Energy Efficient Conditional Broadcasting for Mobile Ad hoc Networks
}

\author{
Shaik Jaffar \\ Associate professor, \\ Madina Engineering college, kadapa, India
}

\author{
M.V Subramanyam, Ph.D \\ Principal, Santhi ram Engineering College, \\ Nandyal, Kurnool Dist, A.P, India
}

\begin{abstract}
Ad-hoc networks include independent self controlled nodes, which use radios for communication. A node can communicate with any other node within its radio frequency range. Pharaonis approaches are the naturally applied strategies by individuals of a group of living beings. In particular, it is from the activities of invertebrates and birds. Although each individual of the swarm has little wisdom and only uses basic principles using neighborhood information acquired from pharaonis approaches such as traveling strategy of birds and ants. In this aspect in our earlier work we suggested a pharaonis approach based routing in mobile ad hoc networks that sent as Pharaonis approach Based Conditional Broadcast Routing (SIBCast). With the inspiration acquired from SIBCast, here within this paper we propose a Pharaonis approach based Energy Efficient Conditional Broadcasting (EECBcast). The aim is to improve transmission functionality alongside energy efficiency that employed for packet transmission. In this paper we use our earlier planned algorithm that inspired from Pharaonis approach to get these features. Within an extensive group of simulator tests, we evaluate this routing algorithm with stateof-the art algorithm, and demonstrate that it gets enhanced performance on a broad range of varied situations and for numerous various assessment measures
\end{abstract}

\section{Keywords}

Manet, Swarm Intelligence, Pharaonis approach, hybrid routing, uniCast routing, ACO

\section{INTRODUCTION}

The Mobile Ad hoc Network is the set of nodes with mobility, which figured connections between them autonomously and enables communication between any two nodes through relay hop nodes without permanent connection setup. This kind of network communication is essential and optimal in situations like military communication and natural catastrophe. In such network states communication between nodes referred as ad hoc routing. A set of protocols that relate ad hoc routing can be found in [1-5]. The protocols such as one that devised in [5] referred as proactive model that let the nodes to exchange routing state sporadically. Another routing strategies such as the models devised in [1-2] referred as reactive, which reacts against to the route request and fixes the route. The routing strategies that are combination of proactive and reactive are referred as hybrid, and this kind of models can be found in [4] [6]. Since any of the routing protocol of these kind are mainly performs on ad hoc structure, the route interruption and improper quality of service factors are quite often. Hence the effective exchange of routing state between relay hops is an essential requirement to avoid frequent route interruptions and also to achieve the better QoS factors.

Pharaonis Approaches (swarm Intelligence based approaches) are proven to be the effective in the direction of route discovery and route maintenance in ad hoc routing protocols.
Few routing strategies like $\mathrm{ABC}$ [19] and antNet [6] are aimed to improve routing in wired networks. These are built on Pharaonis Approach called Ant Colony Optimization [8]. As the factors of node mobility and fluctuated traffic state in Manets, the above said routing models are not fit for Manets. The other factors such as constrained bandwidth, traffic overhead due to overwhelmed control packet traffic in Manets leads to the need of new scalable and effective routing strategies for Manets.

The rest of the paper organized as fallows- section II gives the overview of the proposed EECBcast protocol, route discovery and data transmission strategy in EECBcast, section III explores the simulation and results discussion and that fallowed by the sections IV contains conclusion.

\section{EECBCAST}

1. In the process of route discovery between source node $n_{s}$ and destination node $n_{d}$, the node $n_{s}$ broadcasts the Pharaonis agent $f a_{r e q}$, which targets to trace the route between node $n_{s}$ and node $n_{d}$

2. Due to the process of broadcasting, the target node $n_{d}$ receives multiple $f a_{r e q}$, each represents an unique path between node ' $n_{s}$ ' and ' $n_{d}$ '. Upon receiving a $f a_{r e q}$, the target node $n_{d}$ initiates a pharaonis agent $f a_{\text {res }}$ that transmits through the route discovered by the associated $f a_{r e q}$ and updates the routing and emanation table of each node that visits during the transmission. Each node maintains emanation table such that a pheromone repository of natural swarm agent. The emanation table maintains pharaonis value $p v\left(n_{i}\right)$ of each of its forward relay nodes in the routes between source and target nodes.

3. The failure of the selected route leads to reinitiating of the route discovery process

4. Upon receiving the all $f a_{\text {res }}$ agents by source node $n_{s}$, it initiates optimal route selection by considering quality of service factors such as delay and relay hop count.

\subsection{SIBCast [22]}

SIBCast's style is galvanized by Swarm intelligence based routing strategies of wired networks. It utilizes pharaonis agents that pursue and update emanation tables in an indirect agent interaction for the modification of the surroundings learning method. The packets are routed arbitrarily reliable 
with the learned tables. The SIBCast is distinct from other swarm intelligence approaches since it is hybrid model. It's reactive within the sense that nodes solely group routing info for destinations that they're at present communicating with, whereas it's proactive as a result of nodes try and preserve and improve routing info as long as communiqué goes on. we tend to build a distinction between the trail setup, that is that the reactive apparatus to get initial routing info a couple of destination at the beginning of a session, and path preservation and improvement, that is that the traditional mode of process throughout the course of a session to proactively adapt to network changes. The routing info obtained via indirect agent interaction is unfolded between the nodes of the Manet in relay hop level neighbor info exchange method to supply secondary steerage for the swarm agents. Within the following we offer a broaden explanation of the SIBCAST.

SIBCast's design is stimulated by swarm agent optimized routing algorithms for wired networks. It uses swarm agents which upgrade and follow release tables within an indirect agent conversation concerning the change of the environment learning procedure. Information packages are routed stochastically according to the tables. An essential difference with several other Swarm Agent Enhanced routing calculations is that SIBCAST is a hybrid criteria, in order to cope better with the unique challenges of MANET environments. It really is reactive in the sense that nodes just assemble routing information for locations that they are currently interacting with, although it is proactive because nodes attempt to improve and keep information as long as communication is happening. We produce a distinction between your path set up, that is the reactive mechanism to get initial routing info of a destination at the start of the session, and path maintenance and improvement, which is the standard way of procedure during the course of a program to pro-actively adapt to system adjustments. In the next we supply a concise description of all these components.

\subsection{Pheromone Indicator for EECBcast}

Paths are implicitly outlined by the emanation tables that are kept regionally at every node. An entry $g_{n_{i}}$ of the emanation table $S T_{i}$ at node $n_{i}$ that consider as pheromone point out about the decency of the routing from node $n_{i}$ to via instantaneous node $n_{i}$ contains a price indicating the estimated goodness of going from $n_{i}$ over neighbor $n_{i}$ to reach destination $n_{d}$. This goodness is derived from the grouping of path end-to-end holdup and range of hops. These are commonly used quality measures in Manets. Combining the number of hops with end-to-end delay between immediate node $n_{i}$ to current node $n_{i}$ and destination node $n_{d}$ is a way to swish out presumably giant oscillations within the time estimates congregate by the swarm agents. Since SIBCAST solely maintains info regarding destinations that are active during a communiqué session, and due to continuous modify at neighbor nodes, the filling of the emission tables is dynamic.

\subsection{Route Discovery in EECBcast}

The source node $n_{s}$ determines the path to node $n_{d}$ via broadcasting pharaonis agent for route request $f a_{r e q}$. At each relay hop node that received $f a_{r e q}$, broadcasts the same to their relay hop nodes. This process is recursive for each $f a_{r e q}$ till it received by destination node $n_{d}$. Upon receiving the $f a_{r e q}$, the destination node $n_{d}$ initiates to transmit Routing-path Confirmation Swarm Agent $f a_{r e s}$ that derived from $f a_{r e q} \cdot f a_{r e s}$ Transmits in backward manner through the path that traced by parent $f a_{r e q}$. Upon reaching each node $i$ in the routing path, $f a_{r e s}$ updates pheromone indicator value $g_{n_{i+1}}$ of relay hop node $n_{i+1}$ of the current node $n_{i}$ in the routing path opted by $f a_{r e s}$. The process of updating the pheromone pointer value is as follows:

During the transmission of pharaonis-agent $f a_{r e s}$, it collects the time $t_{n_{i+1} \rightarrow n_{i}}$ taken to reach $n_{i}$ from $n_{i+1}$. The estimated time $t_{n_{i} \rightarrow n_{d}}$ to transmit a data packet from node $n_{i}$ to destination node $n_{d}$ via $\left\{n_{i}, n_{i+1}, n_{i+2} \ldots n_{i+n}\right\}$ is measured using equation (1).

$$
t_{n_{i} \rightarrow n_{d}}=t_{\left(n_{i+n}\right) \rightarrow n_{d}}+\sum_{k=n}^{1} t_{\left(n_{i+k-1}\right) \rightarrow\left(n_{i+k}\right)}
$$

And then pheromone indicator value will be measured using equation (2) and (3) that fallows

$$
\begin{aligned}
& \left(t_{n_{i} \rightarrow n_{d}}\right)^{\prime}=\left[t_{n_{i} \rightarrow n_{d}}\right]^{-1} * 100 \\
& g_{n i}=\frac{\left(t_{n_{i} \rightarrow n_{d}}\right)^{\prime}}{h c_{n_{i} \rightarrow n_{d}}} \ldots \text { (3) }
\end{aligned}
$$

Here in equation (3), $\mathrm{hc}_{n_{i} \rightarrow n_{d}}$ indicates the hop count in path from existing node $n_{i}$ to destination node $n_{d}$ via relay hop node $n_{i}$.

The opposite value of the estimated time $t_{n_{i} \rightarrow n_{d}}$ for a data packet to travel from node $n_{i}$ to destination node $n_{d}$ indicates the optimality of the path between nodes $n_{i}$ to destination node $n_{d}$ via relay node $n_{i}$. Hence the equation (2) is significant. 
Upon receiving swarm agent $f a_{\text {res }}$, the source node $n_{s}$ also updates its emanation table with pheromone pointer value $g_{n i}$ of each neighbor hop $n_{i}$ the $f a_{r e s}$ coming from.

\subsection{Energy Efficient Data transmission and route maintenance in EECBcast}

The routing-path maintenance will be carried out in proactive manner and will be initiated at destination node $n_{d}$. The data transmission and path maintenance strategies explored in fallowing subsections.

\subsubsection{Data Transmission with minimal Energy Usage}

In the process of transmitting data, source and relay hop level node selects the target neighbor relay hop dynamically. Initially source node finds best neighbor $n_{i}$ based on pheromone indicator value of the nodes registered in its emanation table. Opting to a neighbor relay hop $n_{i}$ with best pheromone indicator value $g_{n_{i}}$, transmits data packet to selected neighbor relay hop $n_{i}$. Upon receiving the data packet the neighbor relay hop registers the sender's information in routing cache. The strategy of selecting neighbor relay hop dynamically and transmitting data packet is recursive at each neighbor hop relay node. This process will be halted once the data packet received the destination node $n_{d}$. And as an extension to this process a power conservation mechanism introduced to minimize the energy usage in data transmission that described in section ii that follows.

\subsubsection{Minimal Energy Usage for data transmission in EECBcast}

The nodes are having limited energy and storage capacity, Hence the Energy efficient Swarm Adaptive Hybrid Routing topology has been proposed that saves energy resources. Here in this proposed EECBCAST model the RTS packet takes the energy used for communication by the source node of that RTS. Then the target node of that RTS finds the state of the signal that used to send out RTS .

The proposed EECBCast aims to minimize the energy consumption at nodes in routing topology, which is an essential factor due to the minimal capacity of energy and storage of each node. The strategy of saving energy usage is follows. A node emits an RTS packet to its target relay hop with the information about the energy that spends to communicate. Upon receiving the RTS by target node, it finds the radio frequency used to transmit that RTS

$e_{n_{i+1}}(r)=e_{n_{i}}(r) *\left(\frac{w l_{S}}{4 \pi d}\right)^{2} * \operatorname{str}\left(n_{i}\right) * \operatorname{str}\left(n_{i+1}\right)$

Here $w l_{S}$ is to be the transmitting radio $r$ wavelength, $d$ is distance between $n_{i}$ and $n_{i+1} . \operatorname{str}\left(n_{i}\right)$ is the signal transmission threshold of $r$ at antennas of $n_{i}$ and $\operatorname{str}\left(n_{i+1}\right)$ is the signal receiving threshold of $r_{i}$ at antennas of $n_{i+1} \cdot e_{n_{i}}(r)$ is the maximal frequency possible to spent to transmit $r$ at $n_{i}$ and $e_{n+1}(r)$ is the minimal frequency sufficient to receive the $r$ at $n_{i+1}$. Then the exceeded frequency consumption during $R T S$ transmission between $n_{i}$ and $n_{i+1}$ is

$$
e e_{n_{i}}(r)=e_{n_{i}}(r)-e_{n_{i+1}}(r) \ldots \ldots(5)
$$

Further this $e e_{n_{i}}(r)$ helps to track the actual frequency

$$
\text { levels required between } n_{i} \text { and } n_{i+1} \text { as }
$$

$e_{\text {min }}\left(n_{i} \rightarrow n_{i+1}\right)=i i t *\left(e e_{n_{i}}(r)+m e_{n_{i+1}}(r)\right) \ldots(6)$

The notations used in above equations are as follows:

The ' $e_{\min }\left(n_{i}\right)$ ' indicates minimal frequency at $n_{i}$ used to transmit $r$ towards $n_{i+1}$

The iit is inference influence threshold that used to normalize the frequency against inferences observed during transmission

The ' $m e_{n+1}(r)$ ' represents the least side of the frequency desired at $n_{i+1}$.

Set of routing topologies, which are energy efficiency centric were reviewed in [10]. According to this the variable range transmission energy is the optimal approach among all.

The proposed EECBCast is extended even to minimize the energy consumption by the condition of the radio received in regard to RTS/CTS frames transmission. In regard to achieve the energy efficiency in routing, the route discovery process includes the following strategy. During the route response, each $f a_{\text {res }}$ noticed at the MAC through an unique identifier and grabs its radio frequency state from the physical layer. Upon receiving the $f a_{\text {res }}$ packet by node $n_{i}$ from node $n_{i+1}$, computes $e e_{n_{i}}(r)$ and then stores $e_{\text {min }}\left(n_{i} \rightarrow n_{i+1}\right)$ in its routing table.

The algorithmic representation of the energy efficient data transmission in EECBCast:

Input:

Optimal route $r t$ established during route discovery Source node $n$

Destination node $n_{d}$

Relay hops $\left\{n_{1}, n_{2}, \ldots, n_{i}, n_{i+1}, \ldots n_{i+m}\right\}$ of the route $r t$

Routing Strategy:

- ' $n_{s}$ ' transmits $R T S$ to the relay hop $n_{i}$ of the $r t$, which includes the $e_{\min }\left(n_{s} \rightarrow n_{i}\right)$.

- $\quad$ By receiving $R T S$ sent by $n_{s}$, the $n_{i}$ adjusts its frequency state according to $e_{\min }\left(n_{s} \rightarrow n_{i}\right)$ and 
acknowledges the same to $n_{s}$ through $C T S$ frame.

- $\quad$ By receiving $C T S$ frame sent by $n_{i}$, the source node $n_{s}$ initiates the data transmission under concluded radio frequency.

- The steps 1,2 and 3 continues between any of the two consecutive relay hops of the routing path $r t$

\subsubsection{Routing Path maintenance}

Upon receiving a packet $d p_{i}$, the destination node $n_{d}$ verifies the time $t\left(d p_{i}\right)$ taken by $d p_{i}$ to travel from source node $n_{s}$ to destination node $n_{d}$ and then measures the end to end delay for data packet $d p_{i}$. If end to end delay of $d p_{i}$ is exceeding the delay threshold $\tau$ then it initiates a pharaonis agent $f a_{r e s}$ and transmits towards source node that opts to the path accessed by data packet $d p_{i}$. Hence the ' $f a_{\text {res }}$, performs the process of updating pheromone indicator value $g_{n i}$ at each relay hop level node in the path. This process explored in equations (1), (2) and (3).

\subsubsection{Handling link failures}

The destination node $n_{d}$ initiates swarm agents $f a_{\text {res }}$ to each neighbor relay hop nodes in fixed time intervals. Hence the pheromone indicator values in emanation table of each node will be updated in fixed time interval $\varsigma$.

The pheromone indicator value of any neighbor relay hop $n_{i}$ in emanation table of any node is not valid if time since last update of $g_{n i}$ is greater than time interval $\zeta$. This indicates the link failure between node $i$ and destination node $d$.

\section{EXPERIMENTAL RESULTS}

The experiments aimed to simulate the routing topologies of AODV, CBCast and EECBCast in NS2.The specification of the simulation parameters includes the nodes with in the distance $250 \mathrm{~m}$ is considered as rely hops and in regard to manage the energy consumption, under AODV and CBCast a transmitter used to adjust the transmission energy, but EECBCast relied on proposed variable transmission strategy. The range of the network is set to the area of $1500 \times 1500$ with randomly sprinkled nodes and with indiscriminately selected source and target nodes. The energy levels within the range of 1 to 50 milli-Watts are considered. The standard Poisson distribution is used to set the session arrival rate and exponential allocation is used to fix the session intervals. The node mobility is set as random way point with arbitrarily set pause time. And packet generation is done by CBR topology with the packet creation rate of 100 packets per second. This simulation setup was run for 30 iterations. Since the no energy saving strategy is considered under these simulation topologies, the energy used in the activity of receiving is not considered to be controlled.

We first evaluate the accuracy of the proposed cost model, we then study the performance of route detection for each topology, and finally we consider energy utilization as well as RTS retransmissions in both static and mobile environment.
We compared the energy utilization and the average number of RTS retransmissions of the EECBCAST, CBCAST and basic AODV topologies by varying the following parameters: node count, average size of the data transmission packets, and advent ratio of the connection. The simulation time for each topology is 5 hours. We monitored the total energy utilization of all the packets delivered at target node, the count of delivered packets at target nodes, and the count of retransmissions RTS required for each execution of the simulation. The couple of evaluation metrics that used to evaluate the topologies are:

Energy Utilization per Packet: It is defined by the total energy utilization divided by the total number of packets delivered. This metric indicates the energy efficiency for each topology.

Average RTS Retransmissions required for each Data Packet: It is defined by the total number of RTS retransmissions divided by the total number of packets delivered. The RTS packet is transmitted at the utmost energy usage level and the packet size is very little. The majority of RTS retransmissions are due to collisions, together with the collisions of both RTS messages and data packets. Hence, this metric can indicate the pace of the collision for each topology. Higher collision rate will cause more energy utilization, higher end-to-end delay, and lower throughput.

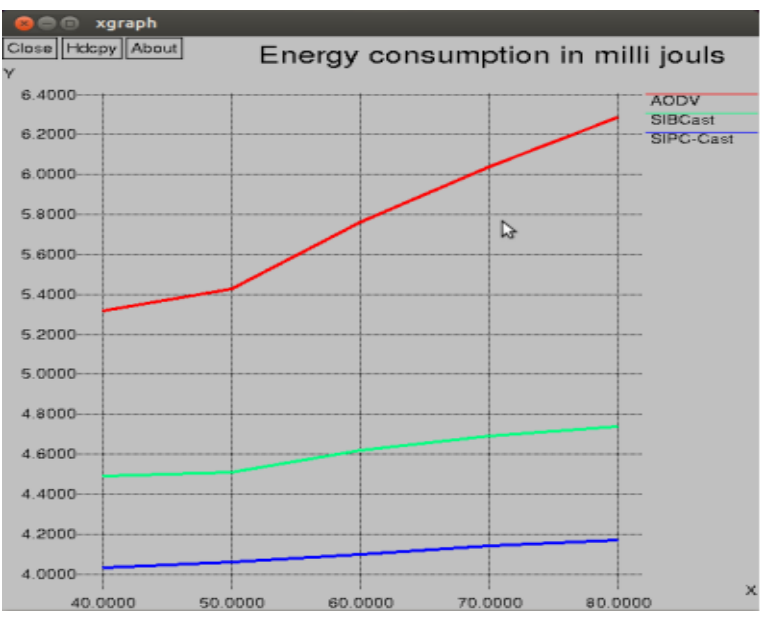

Fig 1: Energy Utilization ratio between EECBcast, SIBCAST and AODV

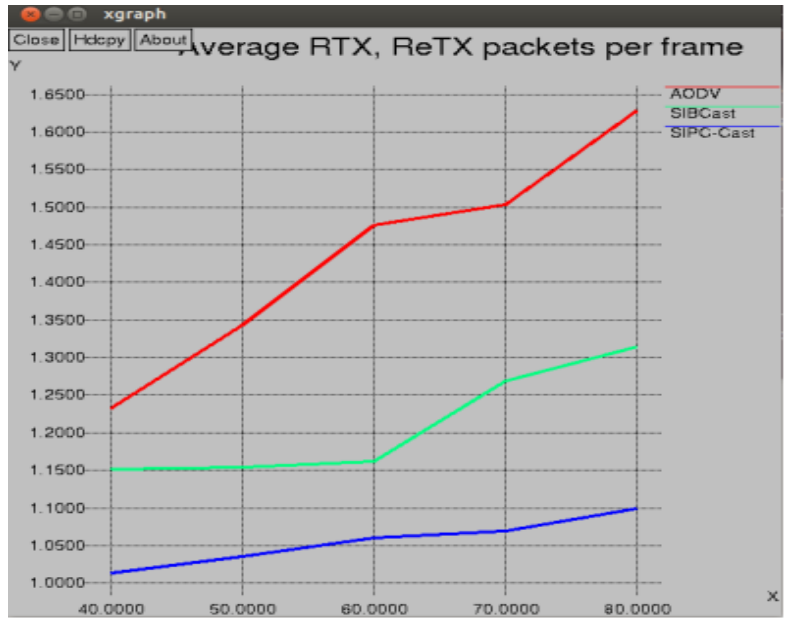

Fig 2: RTX and ReTX packets usage Ratio comparison between AODV, SIBCAST and EECBcast 
The simulation results are shown in Fig. 1 and 2. According to these results, EECBCAST topology performs the best in terms of Energy Utilization per Packet as well as Average RTS Retransmission per Data Packet, followed by CBCAST topology and AODV.

\section{CONCLUSION}

In this paper we have described EECBcast that is an addition to our earlier routing topology SIBCast [22], Pharaonis Approach based Energy Efficient Conditional Broadcasting (EECBcast) topology for MANETs. The algorithm combines reactive and proactive behavior with pharaonis approach adaptation to deal with the QoS related routing challenges of MANETs in an efficient way. This also concern about energy conservation during packet transmission. An efficient relay hop level transmission cost model to more precisely track the energy utilization due to different factors was explored for packet transmission through the route discovered and maintained under SIBCast topology. The simulation studies show that EECBcast topology reduces about $25 \%$ usage of energy used during packet transmission, and is highly adaptive to the surroundings change. In future this topology can be equipped with route overhead endurance mechanism.

\section{REFERENCES}

[1] C. E. Perkins, E. M. Royer, and S. R. Das, "Ad hoc OnDemand Distance Vector (AODV)Routing, Internet Draft (draft-ietf-manet-aodv-09.txt)," November 2001, work in Progress.

[2] D. B. Johnson, D. A. Maltz, Y.-C. Hu, and J. G. Jetcheva, "The Dynamic Source Routing Protocol for Mobile Ad Hoc Networks (DSR), Internet Draft (draft-ietf-manetdsr-07.txt)," February 212002

[3] C. Perkins and P. Bhagwat, "Highly dynamic destinationsequenced distance-vector routing (DSDV) for mobile computers," in ACM SIGCOMM'94 Conference on Communications Architectures, Protocols and Applications, 1994, pp. 234-244

[4] Z. J. Haas, M. R. Pearlman, and P. Samar, "The Zone Routing Protocol (ZRP) for Ad Hoc Networks," July 2002, iETF Internet Draft, draft-ietfmanet- zone-zrp04.txt.

[5] C. Adjih, T. Clausen, P. Jacquet, A. Laouiti, P. Minet, P. Muhlethaler, A. Qayyum, and L. Viennot, "Optimized Link State Routing Protocol," INRIA Rocquencourt," Internet Draft (draft-ietf-manet-olsr-09.txt), April 15 2003, work in Progress.

[6] V. Ramasubramaniam, Z. J. Haas, and E. G. Sirer, "SHARP: A Hybrid Adaptive Routing Protocol for Mobile Ad Hoc Networks," in The ACM Symposium on Mobile Adhoc Networking and Computing (MobiHoc 2003), Annapolis, Maryland, June 1-3 2003.

[7] E. Bonabeau, M. Dorigo, and G. Theraulaz, Pharaonis approach - From Natural to Artificial Systems. New York: Oxford University Press, 1999.

[8] J. P. Sterbenz, R. Krishnan, R. R. Hain, A. W. Jackson, D. Levin, R. Ramanathan, and J. Zao, "Survivable Mobile Wireless Networks: Issues, Challenges, and Research Directions," BBN Technologies, Cambridge, MA, September 2002.

[9] J. S. Baras and H. Mehta, "A Probabilistic Emergent Routing Algorithm for Mobile Ad hoc Networks," in
WiOpt'03: Modeling and Optimization in Mobile, Ad Hoc and Wireless Networks, March 3-5, 2003.

[10]G. D. Caro and M. Dorigo, "AntNet: A Mobile Agents Approach to Adaptive Routing," Universite Libre de Bruxelles, Belgium, Tech. Rep. IRIDIA/97-12, 1997.

[11]D. Camara and A. A. F. Loureiro, "A GPS/Ant-Like Routing Algorithm for Ad Hoc Networks," in IEEE Wireless Communications and Networking Conference (WCNC?00), Chicago, IL, September 2000.

[12]M. Heissenbttel and T. Braun, "Ants-Based Routing in Large Scale Mobile Ad-Hoc Networks," University of Bern, Tech. Rep., 2003

[13]M. Gunes, U. Sorges, and I. Bouazizi, "ARA - The AntColony Based Routing Algorithm for MANETs," in International Conference on Parallel Processing Workshops (ICPPW'02), Vancouver, B.C., Canada, August 18-21 2001.

[14]F. Ducatelle, G. Di Caro, and L. M. Gambardella, "Using Ant Agents to Combine Reactive and Proactive Strategies for Routing in Mobile Ad- Hoc Networks," International Journal of Computational Intelligence and Applications, Special Issue on Nature-Inspired Approaches to Networks and Telecommunications, 2005, to appear. Also Technical Report IDSIA 28-04

[15]G. Di Caro, F. Ducatelle, and L. M. Gambardella, "AntHocNet: an ant-based hybrid routing algorithm for mobile ad hoc networks," in In Proceedings of PPSN VIII - Eight International Conference on Parallel Problem Solving from Nature, ser. Lecture Notes in Computer Science, no. 3242. Birmingham, UK: Springer-Verlag, Sept. 2004, best paper award.

[16]M. Dorigo, G. D. Caro, and L. M. Gambardella, "Ant Algorithms for Discrete Optimization," Universite Libre de Bruxelles, Tech. Rep. IRIDIA/98-10, 1999.

[17]E.M. Royer and C.-K. Toh. A review of current routing protocols for ad hoc mobile wireless networks. IEEE Personal Communications, 1999.

[18]Scalable Network Technologies, Inc., Culver City, CA, USA. Qualnet Simulator, Version 3.6, 2003. http://stargate.ornl.gov/trb/tft.html.

[19]R. Schoonderwoerd, O. Holland, J. Bruten, and L. Rothkrantz. Ant-based load balancing in telecommunications networks. Adaptive Behavior, 5(2):169-207, 1996

[20]R.S. Sutton and A.G. Barto. Reinforcement Learning: An Introduction. MIT Press, 1998.

[21]G. Theraulaz and E. Bonabeau. A brief history of stigmergy. Artificial Life, Special Issue on Stigmergy, 5:97-116, 1999.

[22] Shaik Jaffar, Dr. M.V. Subramanyam; "SIBCast: Pharaonis approach based broadcasting for Mobile Ad hoc Networks "; International Journal of Scientific \& Engineering Research, Volume 4, Issue 11, November2013 1180, ISSN 2229-5518, IJSER (C) 2013, http://www.ijser.org.

[23]Outay, F.; Vèque, V.; Bouallègue, R.; Inst. of Fundamental Electron., Univ. Paris-Sud 11, Orsay, France This paper appears in: 2010 IEEE 29th 

International Performance Computing and
Communications Conference (IPCCC)

\section{AUTHOR'S PROFILE}

S.Jaffar M.Tech, M.I.E, M.I.S.T.E ( Ph.D) Present working as Associate Professor Head of ECE Department at Madina Engineering College Kadapa YSR District Kadapa A.P

Dr.M.V.Subramanyam received his B.E degree in 1989, M. Tech degree in 1997 and PhD degree in 2007. Presently he is working as a 'Principal' and 'Professor' of 'Electronics and Communication Engineering' department of Santhi Ram
Engineering College, Nandyal, Kurnool district, Andhra pradesh, India. He is having more than 23 years of teaching experience. His areas of interest are Adhoc Networks, Computer Networks, Advanced Communications, Signal Processing, Image processing, Embedded Systems and Microcontrollers. He has published more than 40 papers in national and international, conferences and international journals. He is a life member of ISTE, IEEE, IETE, IEI and KDTFM.

Dr.M.V Sbramanyam Principal Shanthi Ram Engineering College Nandyal Kurnool District A.P 